\title{
ESTIMATES FOR THE PROBABILITY THAT A SYSTEM OF RANDOM EQUATIONS IS SOLVABLE IN A GIVEN SET OF VECTORS OVER THE FIELD GF(3)
}

UDC 519.21

\author{
V. I. MASOL AND L. O. ROMASHOVA
}

\begin{abstract}
Let $P_{n}$ be the probability that a second order system of nonlinear random equations over the field GF(3) has a solution in a given set of vectors, where $n$ is the number of unknowns in the system. A necessary and sufficient condition is found for $P_{n} \rightarrow 0$ as $n \rightarrow \infty$. Some rates of convergence to zero are found and some applications are described.
\end{abstract}

\section{Setting of the Problem. Statement of main Results}

Let

$$
\sum_{1 \leq j_{1}<j_{2} \leq n} a_{j_{1} j_{2}}^{(\mu)} x_{j_{1}} x_{j_{2}}=\mathbf{0}, \quad \mu \in J
$$

be a system of nonlinear random equations of the second order considered over the field $\mathbf{G F}(3)$, where $\sum_{3}$ denotes the summation in the field $\mathbf{G F}(3)$ and where $J=\{1, \ldots, T\}$ and $T=T(n)$. Recall that $\mathbf{G F}(3)$ contains only three elements.

We assume that system (11) satisfies the following condition:

(A) the coefficients $a_{j_{1} j_{2}}^{(\mu)}, 1 \leq j_{1}<j_{2} \leq n, \mu \in J$, are independent random variables assuming values in the field $\mathbf{G F}(3)$ according to the distribution

$$
\mathrm{P}\left\{a_{j_{1} j_{2}}^{(\mu)}=\mathbf{1}\right\}=\mathrm{P}\left\{\mathbf{a}_{\mathbf{j}_{1} \mathbf{j}_{\mathbf{2}}}^{(\mu)}=\mathbf{2}\right\}=\mathbf{p}_{\mu}, \quad \mathrm{P}\left\{\mathbf{a}_{\mathbf{j}_{1} \mathbf{j}_{\mathbf{2}}}^{(\mu)}=\mathbf{0}\right\}=\mathbf{1}-\mathbf{2} \mathbf{p}_{\mu} .
$$

Let $V_{n}$ be the family of all $n$-dimensional vectors $\bar{x}, \bar{x}=\left(x_{1}, x_{2}, \ldots, x_{n}\right)$ whose coordinates belong to the field $\mathbf{G F}(3)$, and let $V_{n}^{\prime}=V_{n} \backslash\{\bar{x}:|\bar{x}| \leq 1\}$, where $|\bar{x}|$ denotes the number of nonzero coordinates of the vector $\bar{x}$.

Let $\bar{x}^{(1)}$ and $\bar{x}^{(2)}$ be two arbitrary vectors, where $\bar{x}^{(q)} \in V_{n}, \bar{x}^{(q)}=\left(x_{1}^{(q)}, \ldots, x_{n}^{(q)}\right)$, $q=1,2$. Let $i_{c_{1} c_{2}}, c_{1}, c_{2} \in \mathbf{G F}(3)$, denote the number of pairs $\left(c_{1}, c_{2}\right)$ among $n$ possible pairs $\left(x_{j}{ }^{(1)}, x_{j}{ }^{(2)}\right), 1 \leq j \leq n$.

Let $i=i_{\mathbf{0 1}}+i_{\mathbf{0 2}}$ and $l=i_{\mathbf{1 0}}+i_{\mathbf{2 0}}$.

By $M_{n}$, we denote the maximal subset of the set $V_{n}^{\prime}$ (with respect to the inclusion) with the property that arbitrary vectors $\bar{x}^{(1)}, \bar{x}^{(2)} \in V_{n}^{\prime}$ belong to $M_{n}$ if and only if

$$
i+l \geq 1 \text {. }
$$

For example, if $n=3$, then

$$
M_{3}=\{(\mathbf{1}, \mathbf{1}, \mathbf{0}),(\mathbf{1}, \mathbf{0}, \mathbf{2}),(\mathbf{0}, \mathbf{2}, \mathbf{2}),(\mathbf{1}, \mathbf{2}, \mathbf{1})\} .
$$

Key words and phrases. System of nonlinear random equations, probability that a system is solvable, rate of convergence, a field containing three elements. 
Let $\theta_{n}$ be a random variable equal to the number of solutions of system (1) that belong to the set $M_{n}$.

In what follows we assume that the probability $p_{\mu}$ varies in such a way that

$$
\frac{c \ln n}{n} \leq p_{\mu} \leq \frac{1}{2}-\frac{c \ln n}{n}
$$

where $\ln 3 / \ln 2<a_{1} \leq c=c(n) \leq a_{2}<\infty$ and where $\left\{a_{r}: r=1,2, \ldots\right\}$ is a sequence of bounded positive constants.

Theorem 1.1. Assume that conditions (A) and (3) hold. Then

$$
\mathrm{P}\left\{\theta_{n}>0\right\}=o(1), \quad n \rightarrow \infty,
$$

if and only if

$$
T=n \frac{\ln 2}{\ln 3}+A_{n}
$$

where $A_{n} \rightarrow \infty$ as $n \rightarrow \infty$.

Remark 1.1. The existence of solutions belonging to a given set of vectors for a system of equations is considered in [2] for different right hand sides. In [1], special solutions of a homogeneous system of linear random equations over a finite field are studied and the study of special solutions for the random linear inclusion is considered.

Theorem 1.2. Let conditions (A), (3), and (5) hold. Assume that the parameters $\varepsilon_{1}$, $\varepsilon_{1} \in(0,1)$, and $c$ vary in such a way that

$$
0<\gamma_{1} \leq \varepsilon_{1} c \leq \gamma_{0}<\frac{4}{3}\left(1-\frac{\ln 3}{c \ln 2}\right)
$$

where $\gamma_{0}$ and $\gamma_{1}$ are fixed numbers.

Then there exist a real number $\varepsilon_{2} \in(0,1)$ and natural number $n_{0}=n_{0}\left(\varepsilon_{1}, \varepsilon_{2}, c\right)$ such that $\mathrm{P}\left\{\theta_{n}>0\right\} \leq Z_{1}$ for $n \geq n_{0}$, where

$$
\begin{aligned}
Z_{1}= & \sum_{t=2}^{\left[\sqrt{\frac{\varepsilon_{1} n}{\ln n}}\right]} \frac{1}{t !}\left(\frac{1}{n^{c \frac{\ln 2}{\ln 3}\left(1-\frac{\ln 3}{c \ln 2}-\frac{3}{4} \gamma_{0}\right)}}\right)^{t}\left(\frac{1}{\left.n^{c \frac{A_{n}}{n}\left(1-\frac{3}{4} \gamma_{0}+\frac{3}{4} c \sqrt{\frac{\varepsilon_{1} \ln n}{n}}+\frac{3}{4} c \frac{\ln 2}{\ln 3} \frac{\sqrt{\varepsilon_{1} n \ln n}}{A_{n}}\right.}\right)}\right)^{t} \\
& +2^{n \sigma\left(\varepsilon_{2}\right)}\left(\frac{1}{3}+\frac{2}{\left.3 e^{\frac{3}{2} \gamma_{1}\left(1+\sqrt{\frac{\ln 2}{\ln 3}+A_{n}}\right.}\right)}\right)^{A_{n}} \\
& +\left(\frac{\exp \left\{\frac{2}{n^{\frac{3}{2} n c \varepsilon_{2}^{2}\left(1+\frac{1}{\varepsilon_{2} n}\right)}}\right\}}{3}\right)^{A_{n}} \exp \left\{\frac{2 \ln 2}{n^{\frac{3}{2} n c \varepsilon_{2}^{2}\left(1+\frac{1}{\varepsilon_{2} n}\right)-1} \ln 3}\right\}
\end{aligned}
$$

and where $\sigma\left(\varepsilon_{2}\right)=-\varepsilon_{2} \log _{2} \varepsilon_{2}-\left(1-\varepsilon_{2}\right) \log _{2}\left(1-\varepsilon_{2}\right)$.

Theorem 1.3. Let conditions (A), (3), and (5) hold. Assume that the parameters $\varepsilon_{1}$ and c vary in such a way that

$$
0<\beta_{1} \leq \varepsilon_{1} c \leq \beta_{0}<\frac{4}{3}\left(1-\frac{(1+\alpha) \ln 3}{c \ln 2}\right),
$$

where $\alpha, \beta_{0}$, and $\beta_{1}$ are fixed numbers such that $\alpha>0$ and

$$
\alpha+\frac{3}{4} \beta_{0}<1-\frac{\ln 3}{a_{1} \ln 2} .
$$


Then, given an arbitrary fixed real number $\varepsilon_{2}, \varepsilon_{2} \in(0,1)$, there exists a positive integer number $n_{0}, n_{0}=n_{0}\left(\varepsilon_{1}, \varepsilon_{2}, c\right)$, such that $\mathrm{P}\left\{\theta_{n}>0\right\} \leq Z_{2}$ for $n \geq n_{0}$, where

$$
Z_{2}=\frac{e}{n^{2 \alpha}}+2^{n \sigma\left(\varepsilon_{2}\right)}\left(\frac{1}{3}+\frac{2}{3 e^{\frac{3}{2} \beta_{1}}}\right)^{n \frac{\ln 2}{\ln 3}}+\left(\frac{\exp \left\{\frac{2}{n^{\frac{3}{2} n c \varepsilon_{2}^{2}}}\right\}}{3}\right)^{A_{n}} \exp \left\{\frac{2 \ln 2}{n^{\frac{3}{2} n c \varepsilon_{2}^{2}-1} \ln 3}\right\}
$$

Remark 1.2. The upper bound $Z_{1}\left(Z_{2}\right)$ approaches 0 as $n \rightarrow \infty$ under the assumptions of Theorem 1.2 (Theorem 1.3).

2. The FIRST TWO FACTORIAL MOMENTS OF THE RANDOM VARIABLE $\theta_{n}$

Lemma 2.1. If condition (A) holds, then

$$
\mathrm{E} \theta_{n}=3^{-T} \sum_{t=2}^{n}\left(\begin{array}{l}
n \\
t
\end{array}\right) Q_{t}
$$

where

$$
Q_{t}=\prod_{\mu=1}^{T}\left(1+2\left(1-3 p_{\mu}\right)^{\left(\begin{array}{l}
t \\
2
\end{array}\right)}\right)
$$

Proof. Let the symbol $\xi(\bar{x})$ stand for the indicator of the random event that the vector $\bar{x}$, $\bar{x} \in M_{n}$, is a solution of system (11). Condition (A) implies that

$$
\mathrm{E} \theta_{n}=\sum_{\bar{x}: \bar{x} \in M_{n}} \mathrm{E} \xi(\bar{x})=\sum_{\bar{x}: \bar{x} \in M_{n}} \prod_{\mu=1}^{T} \mathrm{P}\left(\sum_{1 \leq j_{1}<j_{2} \leq n} a_{j_{1} j_{2}}^{(\mu)} x_{j_{1}} x_{j_{2}}=\mathbf{0}\right)
$$

Denote by $t$ the number of nonzero coordinates of an arbitrary fixed vector $\bar{x} \in M_{n}$. We will need the following relation:

$$
\mathrm{P}\{\xi=a\}=\frac{1}{3}-\frac{1}{3}\left(1-3 p^{*}\right)^{k}, \quad a \in \mathbf{G F}(3), a \neq \mathbf{0}
$$

where $\xi=\xi_{1}+{ }_{3} \cdots+{ }_{3} \xi_{k}$ (see [2]). Here $\xi_{1}, \ldots, \xi_{k}, 1 \leq k<\infty$, are independent identically distributed random variables such that $\mathrm{P}\left\{\xi_{s}=\bar{a}\right\}=p^{*}, a \in \mathbf{G F}(3), a \neq \mathbf{0}$, and $\mathrm{P}\left\{\xi_{s}=\mathbf{0}\right\}=1-2 p^{*}, s=1, \ldots, k$. The symbol $+_{3}$ denotes the summation in the field $\mathbf{G F}(3)$.

Using relation (9), we obtain

$$
\prod_{\mu=1}^{T} \mathrm{P}\left(\sum_{1 \leq j_{1}<j_{2} \leq n} a_{j_{1} j_{2}}^{(\mu)} x_{j_{1}} x_{j_{2}}=\mathbf{0}\right)=3^{-T} Q_{t}
$$

The total number of vectors of the set $M_{n}$ that have $t$ nonzero coordinates is equal to the binomial coefficient $\left(\begin{array}{l}n \\ t\end{array}\right)$. Thus, with the help of relation (10), equality (8) can be rewritten in the form of (타).

Let $I=\left\{i_{\mathbf{0 1}}, i_{\mathbf{0 2}}, i_{\mathbf{1 0}}, i_{\mathbf{2 0}}, i_{\mathbf{1 1}}, i_{\mathbf{2 2}}, i_{\mathbf{1 2}}, i_{\mathbf{2 1}}\right\}$.

Lemma 2.2. If condition (A) holds, then

$$
\mathrm{E} \theta_{n}^{[2]}=9^{-T} \sum_{t=3}^{n}\left(\begin{array}{l}
n \\
t
\end{array}\right) \sum_{i+l+h=t} \frac{t !}{h ! i ! l !} Q_{t}^{*},
$$

where

$$
Q_{t}^{*}=\prod_{\mu=1}^{T}\left(1+2\left(\sum_{r=1}^{4}\left(1-3 p_{\mu}\right)^{\Gamma^{(r)}}\right)\right)
$$


The summation $\sum$ above is considered with respect to all indices $i, l$, and $h$ such that $i+l+h=t, t-i \geq 2, t-l \geq 2$, and $i+l \geq 1$; the parameters $\Gamma^{(r)}, r=1, \ldots, 4$, are defined by the equalities

$$
\begin{gathered}
\Gamma^{(1)}=\left(\begin{array}{l}
l \\
2
\end{array}\right)+\left(\begin{array}{l}
i \\
2
\end{array}\right)+(i+l)(t-l-i), \\
\Gamma^{(2)}=\left(\begin{array}{c}
t-l \\
2
\end{array}\right), \\
\Gamma^{(3)}=\left(\begin{array}{c}
t-i \\
2
\end{array}\right), \\
\Gamma^{(4)}=\left(\begin{array}{l}
l \\
2
\end{array}\right)+\left(\begin{array}{l}
i \\
2
\end{array}\right)+\left(\begin{array}{c}
t-l-i \\
2
\end{array}\right)+(i+l)(t-l-i),
\end{gathered}
$$

respectively.

Proof. Condition (A) together with the equality $\mathrm{E} \theta_{n}^{[2]}=\mathrm{E} \theta_{n}\left(\theta_{n}-1\right)$ and representation $\theta_{n}=\sum_{\bar{x}: \bar{x} \in M_{n}} \xi(\bar{x})$ implies that

$$
\mathrm{E} \theta_{n}^{[2]}=\sum_{1} \mathrm{E} \xi\left(\bar{x}^{(1)}\right) \xi\left(\bar{x}^{(2)}\right),
$$

where the summation $\sum_{1}$ is considered with respect to all pairs of vectors $\left(\bar{x}^{(1)}, \bar{x}^{(2)}\right)$ such that $\bar{x}^{(q)} \in M_{n}, q=1,2$, and $\bar{x}^{(1)} \neq \bar{x}^{(2)}$. With the help of equality (17) we find that

$$
\begin{aligned}
\mathrm{E} \theta_{n}^{[2]} & =\sum_{1} \prod_{\mu=1}^{T} \mathrm{P}\left\{\bigcup\left\{A^{(\mu)}\left(\bar{x}^{(k)}\right)=y_{k}, A^{(\mu)}\left(\bar{x}^{(1)}, \bar{x}^{(2)}\right)=y_{12}, k=1,2\right\}\right\} \\
& =\sum_{1} \prod_{\mu=1}^{T} \sum_{2} \mathrm{P}\left\{A^{(\mu)}\left(\bar{x}^{(1)}, \bar{x}^{(2)}\right)=y_{12}\right\} \prod_{k=1,2} \mathrm{P}\left\{A^{(\mu)}\left(\bar{x}^{(k)}\right)=y_{k}\right\},
\end{aligned}
$$

where the symbol $\bigcup\left(\sum_{2}\right)$ means the union (summation) corresponding to all solutions of the following system of the two equations $y_{1}+3 y_{12}=\mathbf{0}$ and $y_{2}+_{3} y_{12}=\mathbf{0}$ over the field $\mathbf{G F}(3)$, where

$$
A^{(\mu)}\left(\bar{x}^{(1)}, \bar{x}^{(2)}\right)=\sum_{\omega \in E^{(12)}} a_{\omega}^{(\mu)}, A^{(\mu)}\left(\bar{x}^{(q)}\right)=\sum_{\omega \in E^{(q)}} a_{\omega}^{(\mu)}, \quad q=1,2,
$$

for $\mu \in J$, and where

$$
\begin{gathered}
E^{(12)}=\left\{\left(j_{1}, j_{2}\right), 1 \leq j_{1}<j_{2} \leq n: x_{j_{1}}^{(q)} x_{j_{2}}^{(q)} \neq \mathbf{0}, q=1,2\right\}, \\
E^{(q)}=\left\{\left(j_{1}, j_{2}\right), 1 \leq j_{1}<j_{2} \leq n: x_{j_{1}}^{(q)} x_{j_{2}}^{(q)} \neq \mathbf{0}, x_{j_{1}}^{\left(q^{*}\right)} x_{j_{2}}^{\left(q^{*}\right)}=\mathbf{0}\right\},
\end{gathered}
$$

$q \in\{1,2\}, q^{*} \in\{1,2\}, q^{*} \neq q$.

Let $\gamma^{(1)}, \gamma^{(2)}$, and $\gamma^{(3)}$ be the number of elements of the sets $E^{(1)}, E^{(2)}$, and $E^{(12)}$, respectively.

Put

$$
\begin{gathered}
\Gamma^{(1)}=\gamma^{(1)}+\gamma^{(2)}, \quad \Gamma^{(2)}=\gamma^{(2)}+\gamma^{(3)}, \\
\Gamma^{(3)}=\gamma^{(1)}+\gamma^{(3)}, \quad \Gamma^{(4)}=\gamma^{(1)}+\gamma^{(2)}+\gamma^{(3)} .
\end{gathered}
$$

Considering condition (A) and using equality (9), relation (18) can be rewritten as follows:

$$
\mathrm{E} \theta_{n}^{[2]}=9^{-T} \sum_{1} \prod_{\mu=1}^{T}\left(1+2\left(\sum_{r=1}^{4}\left(1-3 p_{\mu}\right)^{\Gamma^{(r)}}\right)\right)
$$


For arbitrary vectors $\bar{x}^{(1)}, \bar{x}^{(2)} \in M_{n}$, denote by $t$ the total number of pairs $\left(c_{1}, c_{2}\right)$, $\left(c_{3}, \mathbf{0}\right),\left(\mathbf{0}, c_{4}\right)$ among $n$ possible pairs $\left(x_{j}{ }^{(1)}, x_{j}{ }^{(2)}\right), 1 \leq j \leq n$, with the property that $c_{1} c_{2} c_{3} c_{4} \neq \mathbf{0}$ for all $c_{1}, c_{2}, c_{3}, c_{4} \in \mathbf{G F}(3)$. Then $t=i+l+h$ and the total number of pairs of vectors $\left(\bar{x}^{(1)}, \bar{x}^{(2)}\right)$ for which equality (21) holds is found from the following equation:

$$
\sum_{i+l+h=t} \frac{n !}{h ! i ! l !(n-t) !}=\left(\begin{array}{l}
n \\
t
\end{array}\right) \sum_{i+l+h=t} \frac{t !}{h ! i ! l !} .
$$

The summation $\sum_{1}$ on the right hand side of (21) means the summation over all pairs of vectors $\left(\bar{x}^{(1)}, \bar{x}^{(2)}\right)$ such that $\bar{x}^{(1)} \neq \bar{x}^{(2)}, \bar{x}^{(q)} \in M_{n}, q=1,2$, and is equivalent to the summation with respect to all parameters $i, l$, and $h$ written on the right hand side of (11). Note that the inequalities $t-i \geq 3, t-l \geq 3$, and $i+l \geq 1$ imply that $\left|\bar{x}^{(1)}\right| \geq 1$, $\left|\bar{x}^{(2)}\right| \geq 1$, and $\bar{x}^{(1)} \neq \bar{x}^{(2)}$, respectively.

Next we check equality (13). First, we find some explicit expressions for the parameters $\gamma^{(1)}$ and $\gamma^{(2)}$.

Our current goal is to show that

$$
\gamma^{(1)}=\left|E^{(1)}\right|=\left(\begin{array}{l}
l \\
2
\end{array}\right)+l(t-l-i) .
$$

Indeed, we represent $\gamma^{(1)}$ as a sum of two terms, namely

$$
\gamma^{(1)}=\left|E_{1}^{(1)}\right|+\left|E_{2}^{(1)}\right|
$$

where

$$
\begin{gathered}
E_{1}^{(1)}=\left\{\left(j_{1}, j_{2}\right), 1 \leq j_{1}<j_{2} \leq n: x_{j_{1}}^{(1)}, x_{j_{2}}^{(1)} \neq \mathbf{0} ; x_{j_{1}}^{(2)}=x_{j_{2}}^{(2)}=\mathbf{0}\right\}, \\
E_{2}^{(1)}=\left\{\left(j_{1}, j_{2}\right), 1 \leq j_{1}<j_{2} \leq n: x_{j_{1}}^{(1)}, x_{j_{2}}^{(1)} \neq \mathbf{0} ; x_{j}^{(2)}=\mathbf{0}, x_{j^{*}}^{(2)} \neq \mathbf{0}\right\},
\end{gathered}
$$

$j \in\left\{j_{1}, j_{2}\right\}, j^{*} \in\left\{j_{1}, j_{2}\right\}, j \neq j^{*}$.

Since the sum $i_{\mathbf{1 0}}+i_{\mathbf{2 0}}$ means the total number of nonzero coordinates of the vector $\bar{x}^{(1)}$ corresponding to the nonzero coordinates of the vector $\bar{x}^{(2)}$ and since $i_{\mathbf{1 1}}+i_{\mathbf{2 2}}+i_{\mathbf{1 2}}+i_{\mathbf{2 1}}$ means the number of the nonzero coordinates in the vector $\bar{x}^{(1)}$ corresponding to the nonzero coordinates of the vector $\bar{x}^{(2)}$, we find

$$
\begin{gathered}
\left|E_{1}^{(1)}\right|=\left(\begin{array}{l}
l \\
2
\end{array}\right), \\
\left|E_{2}^{(1)}\right|=l(t-l-i) .
\end{gathered}
$$

Taking into account equalities (23) $-(25)$ we obtain (22).

Similarly we have

$$
\gamma^{(2)}=\left|E^{(2)}\right|=\left(\begin{array}{l}
i \\
2
\end{array}\right)+i(t-l-i) .
$$

Thus (19), (22), and (26) imply relation (13). Finally, the definition of the set $E^{(12)}$ proves that

$$
\gamma^{(3)}=\left|E^{(12)}\right|=\left(\begin{array}{c}
t-i-l \\
2
\end{array}\right)
$$

Now (19), (26), and (27) imply equality (14). Then we derive (15) from (20), (23), and (27). Using (20), (23), (26), and (27) we get equality (16). 
Remark 2.1. We see from the proof of Lemma 2.2 that the number $i+l+h$ (see equality (11D) is equal to the sum of elements of the set $I$. In particular, $h=i_{\mathbf{1 1}}+i_{\mathbf{2 2}}+i_{\mathbf{1 2}}+i_{\mathbf{2 1}}$ and $l=i_{\mathbf{1 0}}+i_{\mathbf{2 0}}$.

\section{Auxiliary Results}

Lemma 3.1. If condition (A) holds and

$$
p_{\mu} \leq \frac{1}{2}-v
$$

where $0<v \leq \frac{1}{2}$ and $\mu \in J$, then

$$
\mathrm{E} \theta_{n}>0
$$

for an arbitrary $n \geq 2$.

Proof. In view of (6) and (7), relation (29) follows if

$$
Q_{t}>0, \quad n \geq 2 .
$$

To prove inequality (30) we represent the product $Q_{t}$ defined by equality (77) as follows:

$$
Q_{t}=\prod_{r=1}^{3} Q_{t ; r},
$$

where $Q_{t ; r}$ means the product of all factors on the right hand side of equality (7) for which the parameter $\mu$ belongs to the set $W_{r}, r=1,2,3$. Here

$$
\begin{gathered}
W_{1}=\left\{\mu, 1 \leq \mu \leq T: p_{\mu} \leq \frac{1}{3}\right\}, \\
W_{2}=\left\{\mu, \quad 1 \leq \mu \leq T: \frac{1}{3}<p_{\mu} \leq \frac{1}{2}-v,\left(\begin{array}{l}
t \\
2
\end{array}\right) \text { is even, } t \geq 2\right\}, \\
W_{3}=\left\{\mu, \quad 1 \leq \mu \leq T: \frac{1}{3}<p_{\mu} \leq \frac{1}{2}-v,\left(\begin{array}{l}
t \\
2
\end{array}\right) \text { is odd, } t \geq 2\right\} .
\end{gathered}
$$

Denote by $\eta_{r}$ the number of elements of the set $W_{r}$, that is, $\eta_{r}=\left|W_{r}\right|, r=1,2,3$. Then

$$
\sum_{r=1}^{3} \eta_{r}=T .
$$

The definition of the products $Q_{t ; 1}$ and $Q_{t ; 2}$ implies that

$$
Q_{t ; 1} \geq 1, \quad Q_{t ; 2} \geq 1 \text {. }
$$

Considering condition (28), we find

$$
Q_{t ; 3} \geq(6 v)^{\eta_{3}}
$$

It follows from (31)- (34) that $Q_{t} \geq(6 v)^{\eta_{3}}$, whence we obtain inequality (30) and hence (29) is proved.

Lemma 3.2. Assume that conditions (A) and (3) hold. If

$$
T \leq n \frac{\ln 2}{\ln 3}+m_{0}
$$

as $n \rightarrow \infty$, where $m_{0}$ is a constant, then

$$
Q_{t} \geq a_{3}, \quad n \rightarrow \infty,
$$

for an arbitrary $t \in F$, where

$$
F=\left[\left[\frac{n}{2}\right]-\left[\frac{n}{\ln n}\right] ; n\right]
$$


Proof. Taking into account representation (31) we see that relation (36) follows if, for $t \in F$ and as $n \rightarrow \infty$, there exists a constant $a_{4}$ such that

$$
Q_{t ; r} \geq a_{4}, \quad r=1,2,3 .
$$

Similarly to the proof of inequality (33) we obtain $Q_{t ; 1} \geq 1$ and $Q_{t ; 2} \geq 1$ for $t \in F$, $\mu \in W_{1}$, and $\mu \in W_{2}$ if $n>1$.

Now we check representation (37) for $r=3$. Indeed, taking into account (3) and the inclusion $\mu \in W_{3}$, we prove that, for $t \in F$,

$$
\left(1-3 p_{\mu}\right)^{\left(\begin{array}{l}
t \\
2
\end{array}\right)} \geq-2^{-\frac{n^{2}}{8}(1+o(1))} \exp \left\{-\frac{3}{4} c n(1+o(1)) \ln n\right\}
$$

as $n \rightarrow \infty$, where $c>\ln 3 / \ln 2$.

Using (7), (35), and (38) we get

$$
Q_{t ; 3} \geq a_{5}\left(1-a_{6} 2^{-\frac{n^{2}}{8}(1+o(1))} \exp \left\{-\frac{3}{4} c n(1+o(1)) \ln n\right\}\right)^{n \frac{\ln 2}{\ln 3}}, \quad n \rightarrow \infty .
$$

This implies inequality (37) for $r=1,2,3$. Now relations (31) and (37) prove (36).

Lemma 3.3. Let $b$ and $c$ be fixed integer numbers such that $0<b<c$ and let $\psi_{n}$ be $a$ sequence of integer numbers such that $\psi_{n} / n \rightarrow 0$ as $n \rightarrow \infty$. Then

$$
\left(\begin{array}{c}
n \\
{\left[\frac{b}{c} n\right]-\psi_{n}}
\end{array}\right)<\frac{c^{n} \exp \left\{-\frac{\psi_{n}^{2}}{n}\left(\frac{c^{2}}{2 b(c-b)}+O\left(\frac{\psi_{n}}{n}\right)\right)\right\}}{b^{\frac{b}{c} n-\psi_{n}}(c-b)^{\frac{c-b}{c} n+\psi_{n}}}, \quad n \rightarrow \infty .
$$

Proof. Relation (39) follows from the Stirling formula [4].

Lemma 3.4. Let condition (A) hold and let $t \geq 4$ be an arbitrary number. Then, among the four parameters $\Gamma^{\left(l_{0}\right)}, l_{0}=1, \ldots, 4$, defined by relations (13)-(16), there are at least three parameters $\Gamma^{\left(l_{1}\right)}, \Gamma^{\left(l_{2}\right)}, \Gamma^{\left(l_{3}\right)}, l_{1}, l_{2}, l_{3} \in\{1,2,3,4\}, l_{1} \neq l_{2}, l_{2} \neq l_{3}, l_{1} \neq l_{3}$, such that

$$
\Gamma^{\left(l_{r}\right)} \geq \frac{t}{2}-1, \quad r=1,2,3 .
$$

Moreover, among these three parameters, there exists at least one parameter

$$
\Gamma^{\left(l^{*}\right)}, \quad l^{*} \in\left\{l_{1}, l_{2}, l_{3}\right\},
$$

such that

$$
\Gamma^{\left(l^{*}\right)} \geq\left(\begin{array}{c}
\frac{t}{2} \\
2
\end{array}\right)
$$

Proof. Let $i \geq \frac{t}{2}$. Then, applying relations (13)-(16), we prove that there are at least three parameters $\Gamma^{\left(l_{1}\right)}, \Gamma^{\left(l_{2}\right)}, \Gamma^{\left(l_{3}\right)}, l_{1}, l_{2}, l_{3} \in\{1,2,3,4\}, l_{1} \neq l_{2}, l_{2} \neq l_{3}, l_{1} \neq l_{3}$, such that $\Gamma^{\left(l_{r}\right)} \geq\left(\begin{array}{c}\frac{t}{2} \\ 2\end{array}\right), t \geq 4, r=1,2,3$.

Now let

$$
i<\frac{t}{2}
$$

Consider separately all possible cases.

1) If inequality (40) holds and $l \geq \frac{t}{2}$, then

$$
\Gamma^{(1)} \geq\left(\begin{array}{l}
l \\
2
\end{array}\right) \geq\left(\begin{array}{l}
\frac{t}{2} \\
2
\end{array}\right), \quad \Gamma^{(3)} \geq\left(\begin{array}{l}
l \\
2
\end{array}\right) \geq\left(\begin{array}{l}
\frac{t}{2} \\
2
\end{array}\right), \quad \Gamma^{(4)} \geq \Gamma^{(1)} \geq\left(\begin{array}{l}
\frac{t}{2} \\
2
\end{array}\right)
$$

by (13), (15), and (16); 
2) if inequality (40) holds and $h \geq \frac{t}{2}$, where $t=i+l+h$ and $h=i_{\mathbf{1 1}}+i_{\mathbf{2 2}}+i_{\mathbf{1 2}}+i_{\mathbf{2 1}}$, then we use relations (14), (15), and (16) and obtain the bounds

$$
\Gamma^{(2)} \geq\left(\begin{array}{l}
h \\
2
\end{array}\right) \geq\left(\begin{array}{c}
\frac{t}{2} \\
2
\end{array}\right), \quad \Gamma^{(3)} \geq\left(\begin{array}{l}
h \\
2
\end{array}\right) \geq\left(\begin{array}{c}
\frac{t}{2} \\
2
\end{array}\right), \quad \Gamma^{(4)} \geq\left(\begin{array}{c}
t-l-i \\
2
\end{array}\right)=\left(\begin{array}{l}
h \\
2
\end{array}\right) \geq\left(\begin{array}{c}
\frac{t}{2} \\
2
\end{array}\right) ;
$$

3 ) if inequality (40) holds and $l+h \geq \frac{t}{2}, l \geq 1$, and $h \geq 1$ (the cases where $l \geq \frac{t}{2}$ and $h=0$ or $l=0$ and $h \geq \frac{t}{2}$ are considered in cases 1) and 2) above), then $l h \geq \frac{t}{2}-1$.

Indeed, let $l+h=\beta$, where $\beta \geq \frac{t}{2}$. Then $l h=l(\beta-l) \geq \beta-1 \geq \frac{t}{2}-1$. The inequality $l(\beta-l) \geq \beta-1$ holds, since the function $f(x)=x(\beta-x)$ increases in the interval $[1 ; \beta / 2]$, decreases in the interval $[\beta / 2 ; \beta-1]$, and attains its minimal value at $x=1$ or at $x=\beta-1$ (without loss of generality we assume that $1 \leq x \leq \beta-1$ ).

Therefore (13), (15), (16) and the inequality $l h \geq \frac{t}{2}-1, l+h \geq \frac{t}{2}$ imply that

$$
\begin{gathered}
\Gamma^{(1)} \geq(i+l)(t-l-i)=(i+l) h \geq l h \geq \frac{t}{2}-1, \\
\Gamma^{(3)}=\left(\begin{array}{c}
h+l \\
2
\end{array}\right) \geq\left(\begin{array}{c}
\frac{t}{2} \\
2
\end{array}\right), \quad \Gamma^{(4)} \geq \Gamma^{(1)} \geq \frac{t}{2}-1 .
\end{gathered}
$$

Let $p_{\min }=\min _{1 \leq \mu \leq T} p_{\mu}$. In what follows the symbol $\varepsilon_{q}$ stands for a positive fixed number whose precise value is specified for each appearance of $q, q \geq 1$.

Consider the sums

$$
D_{z}=3^{-T} \sum_{t \in \mathbb{R}_{z}}\left(\begin{array}{l}
n \\
t
\end{array}\right) Q_{t}
$$

where $z=1,2,3$,

$$
R_{1}=\left[2 ;\left[\sqrt{\frac{\varepsilon_{1} n}{\ln n}}\right]\right], \quad R_{2}=\left[\left[\sqrt{\frac{\varepsilon_{1} n}{\ln n}}\right]+1 ;\left[\varepsilon_{2} n\right]\right], \quad R_{3}=\left[\left[\varepsilon_{2} n\right]+1 ; n\right] .
$$

Lemma 3.5. Let conditions (A), (3), and (5) hold. Assume that the parameters $\varepsilon_{1}$ and $c$ are varying in such a way that

$$
\varepsilon_{1} c \leq \gamma_{0}<\frac{4}{3}\left(1-\frac{\ln 3}{c \ln 2}\right) .
$$

Then

$$
\begin{aligned}
& D_{1} \leq \sum_{t=2}^{\left[\sqrt{\frac{\varepsilon_{1} n}{\ln n}}\right]} \frac{1}{t !}\left(\frac{1}{n^{c \frac{\ln 2}{\ln 3}\left(1-\frac{\ln 3}{c \ln 2}-\frac{3}{4} \gamma_{0}\right)}}\right)^{t} \\
& \left.\times\left(\frac{1}{n c \frac{A_{n}}{n}\left(1-\frac{3}{4} \gamma_{0}+\frac{3}{4} c \sqrt{\frac{\varepsilon_{1} \ln n}{n}}+\frac{3}{4} c \frac{\ln 2}{\ln 3} \frac{\sqrt{\varepsilon_{1} n \ln n}}{A_{n}}\right.}\right)\right)^{t} .
\end{aligned}
$$

Proof. Taking into account (3) and (17) we obtain

$$
Q_{t} \leq 3^{T}\left(1-2 p_{\min }\left(\begin{array}{l}
t \\
2
\end{array}\right)+3\left(p_{\min }\left(\begin{array}{l}
t \\
2
\end{array}\right)\right)^{2}\right)^{T}
$$

for $t \in\left[2 ;\left[\sqrt{\frac{\varepsilon_{1} n}{\ln n}}\right]\right]$. It follows from (42) that

$$
D_{1} \leq \sum_{t=2}^{\left[\sqrt{\frac{\varepsilon_{1} n}{\ln n}}\right]} \frac{n^{t}}{t !} \exp \left\{-T t p_{\min }\left(1-\frac{3}{4} \frac{\varepsilon_{1} n}{\ln n}\left(1-\sqrt{\frac{\ln n}{\varepsilon_{1} n}}\right) p_{\min }\right)\right\}
$$

for all $t \in\left[2 ;\left[\sqrt{\frac{\varepsilon_{1} n}{\ln n}}\right]\right]$.

Now conditions (3) and (5) together with inequality (43) imply bound (41). 
Lemma 3.6. Let conditions (A), (3), and (5) hold. Assume that the parameters $\varepsilon_{1}$ and $c$ are varying in such a way that $\varepsilon_{1} c \geq \gamma_{1}>0$. Then there exists a number $\varepsilon_{2}, 0<\varepsilon_{2}<1$, such that

$$
D_{2} \leq 2^{n \sigma\left(\varepsilon_{2}\right)}\left(\frac{1}{3}+\frac{2}{3 e^{\frac{3}{2} \gamma_{1}\left(1+\sqrt{\frac{\ln n}{\varepsilon_{1} n}}\right)}}\right)^{n \frac{\ln 2}{\ln 3}+A_{n}},
$$

where $\sigma\left(\varepsilon_{2}\right)=-\varepsilon_{2} \log _{2} \varepsilon_{2}-\left(1-\varepsilon_{2}\right) \log _{2}\left(1-\varepsilon_{2}\right)$.

Proof. For $t \in\left[\left[\sqrt{\frac{\varepsilon_{1} n}{\ln n}}\right]+1,\left[\varepsilon_{2} n\right]\right]$, we get

$$
Q_{t} \leq\left(1+2 \exp \left\{-3 p_{\min }\left(\begin{array}{c}
{\left[\sqrt{\frac{\varepsilon_{1} n}{\ln n}}\right]+1} \\
2
\end{array}\right)\right\}\right)^{T} .
$$

Now relations (3), (5), and (45) yield

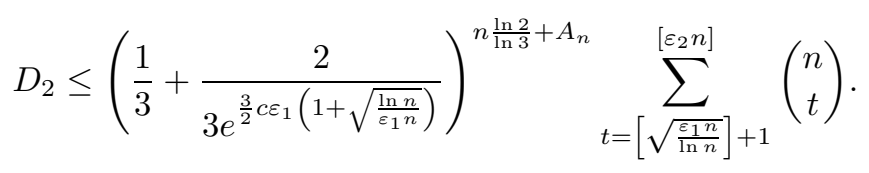

The inequality

$$
\sum_{t=0}^{\left[\varepsilon_{2} n\right]}\left(\begin{array}{l}
n \\
t
\end{array}\right) \leq 2^{n \sigma\left(\varepsilon_{2}\right)}
$$

implies bound (44) in view of inequality (46), where

$$
\sigma\left(\varepsilon_{2}\right)=-\varepsilon_{2} \log _{2} \varepsilon_{2}-\left(1-\varepsilon_{2}\right) \log _{2}\left(1-\varepsilon_{2}\right)
$$

(see [3]).

Lemma 3.7. Let conditions (A), (3), and (5) hold. Then

$$
D_{3} \leq\left(\frac{\exp \left\{\frac{2}{\left.n^{\frac{3}{2} n c \varepsilon_{2}^{2}\left(1+\frac{1}{\varepsilon_{2} n}\right.}\right)}\right\}}{3}\right)^{A_{n}} \exp \left\{\frac{2 \ln 2}{n^{\frac{3}{2} n c \varepsilon_{2}^{2}\left(1+\frac{1}{\varepsilon_{2} n}\right)-1} \ln 3}\right\}
$$

for $\varepsilon_{2}>0$.

Proof. For $t \in\left[\left[\varepsilon_{2} n\right]+1, n\right]$, we have

$$
Q_{t} \leq\left(1+2 \exp \left\{-3 p_{\min }\left(\begin{array}{c}
{\left[\varepsilon_{2} n\right]+1} \\
2
\end{array}\right)\right\}\right)^{T} .
$$

Using (3) and (48), we obtain

$$
D_{3} \leq \frac{2^{n}}{3^{T}} \exp \left\{\frac{2 T}{\exp \left\{\frac{3}{2} c \varepsilon_{2}^{2} n(\ln n)\left(1+\frac{1}{\varepsilon_{2} n}\right)\right\}}\right\} .
$$

Now we derive

$$
D_{3} \leq \frac{2^{n}}{3^{\frac{\ln 2}{\ln 3} n+A_{n}}} \exp \left\{\frac{2 n \frac{\ln 2}{\ln 3}+2 A_{n}}{n^{\frac{3}{2} n c \varepsilon_{2}^{2}\left(1+\frac{1}{\varepsilon_{2} n}\right)}}\right\}
$$

from (5) and (49), whence inequality (47) follows. 


\section{Proof of Theorem 1.1}

Proof. Sufficiency. We show that (5) implies

$$
\mathrm{E} \theta_{n}=o(1), \quad n \rightarrow \infty .
$$

Considering (6) and (7), the expectation $\mathrm{E} \theta_{n}$ can be written as follows:

$$
\mathrm{E} \theta_{n}=\sum_{h=1}^{3} D_{h}
$$

where $D_{1}, D_{2}$, and $D_{3}$ are defined above.

Taking into account representation (51), relation (50) follows from

$$
D_{h}=o(1), \quad n \rightarrow \infty,
$$

for $h=1,2,3$. Using (41), (44), and (47), one easily checks relation (52) for $h=1, h=2$, and $h=3$, respectively.

Now relation (50) follows from (51) and (52). Using (50) and Chebyshev's inequality, we prove (4).

Necessity. Let $\mathrm{P}\left\{\theta_{n}>0\right\} \rightarrow 0$ as $n \rightarrow \infty$. We show that (5) holds. If equality (5) does not hold, then equality (35) holds. Our current goal is to show that there exists a positive constant $C$ such that

$$
\mathrm{P}\left\{\theta_{n}>0\right\} \geq C>0, \quad n \rightarrow \infty .
$$

In other words, relation (53) means that, with a positive probability, there exists a solution that belongs to the set $M_{n}$. First we prove the following upper bounds:

$$
\begin{gathered}
\left(\mathrm{E} \theta_{n}\right)^{-1} \leq a_{5}, \\
\mathrm{E} \theta_{n}^{[2]}\left(\mathrm{E} \theta_{n}\right)^{-2} \leq a_{6}
\end{gathered}
$$

and use them further in the inequality

$$
\mathrm{P}\left\{\theta_{n}>0\right\} \geq\left(\left(\mathrm{E} \theta_{n}\right)^{-1}+\mathrm{E} \theta_{n}^{[2]}\left(\mathrm{E} \theta_{n}\right)^{-2}\right)^{-1}
$$

(see [5]).

Then relations (6) and (29) together with Lemma 3.2 imply that

$$
\left(\mathrm{E} \theta_{n}\right)^{-1} \leq 3^{T} 2^{-n} \delta_{n},
$$

where

$$
\delta_{n} \leq a_{3}^{-1}\left(2^{-n} \sum_{t \in F}\left(\begin{array}{l}
n \\
t
\end{array}\right)\right)^{-1}, \quad n \rightarrow \infty .
$$

Lemma 3.3 with $b=1, c=2$, and $\psi_{n}=[n / \ln n]$ allows one to conclude that

$$
2^{-n} \sum_{t \in F}\left(\begin{array}{l}
n \\
t
\end{array}\right) \rightarrow 1, \quad n \rightarrow \infty,
$$

which together with (35), (57), and (58) proves (54).

Similarly to the proof of (54), we make sure that

$$
\left(3^{T} 2^{-n} \mathrm{E} \theta_{n}\right)^{-1} \leq a_{7}, \quad n \rightarrow \infty .
$$

Next, relation (59) implies that inequality (55) follows from

$$
9^{T} 4^{-n} \mathrm{E} \theta_{n}^{[2]} \leq a_{8}, \quad n \rightarrow \infty .
$$

Considering (11), the left hand side of (60) can be rewritten as follows:

$$
9^{T} 4^{-n} \mathrm{E} \theta_{n}^{[2]}=4^{-n} S\left(n ; Q_{t}^{*}\right),
$$


where

$$
S\left(n ; Q_{t}^{*}\right)=\sum_{t=3}^{n}\left(\begin{array}{l}
n \\
t
\end{array}\right) \sum_{i+l+h=t} \frac{t !}{i ! l ! h !} Q_{t}^{*} .
$$

We represent $S\left(n ; Q_{t}^{*}\right)$ as a sum of two terms $S_{1}\left(n ; Q_{t}^{*}\right)$ and $S_{2}\left(n ; Q_{t}^{*}\right)$, namely

$$
S\left(n ; Q_{t}^{*}\right)=S_{1}\left(n ; Q_{t}^{*}\right)+S_{2}\left(n ; Q_{t}^{*}\right),
$$

where $S_{1}\left(n ; Q_{t}^{*}\right)$ differs from $S\left(n ; Q_{t}^{*}\right)$ by the set of summation on the right hand side of (62) where the indices $i, l$, and $h$ are such that

$$
\Gamma^{(r)} \geq\left(\begin{array}{c}
\varepsilon n \\
2
\end{array}\right)
$$

where $\varepsilon$ is a constant such that $0<\varepsilon<1$, and where $\Gamma^{(r)}, r=1, \ldots, 4$, are defined by equalities (13)-(16). Here $S_{2}\left(n ; Q_{t}^{*}\right)$ is the sum of the rest of the terms in $S\left(n ; Q_{t}^{*}\right)$.

Relations (3), (12), (35), and (64) imply that

$$
S_{1}\left(n ; Q_{t}^{*}\right) \leq a_{9} S_{1}(n ; 1), \quad n \rightarrow \infty .
$$

The inequality $S_{1}(n ; 1) \leq 4^{n}$ together with relation (65) yields

$$
S_{1}\left(n ; Q_{t}^{*}\right) \leq a_{9} 4^{n}
$$

as $n \rightarrow \infty$.

Next we represent the sum $S_{2}\left(n ; Q_{t}^{*}\right)$ as follows:

$$
S_{2}\left(n ; Q_{t}^{*}\right)=\sum_{k=1}^{4} S_{2 ; k}\left(n ; Q_{t}^{*}\right),
$$

where $S_{2 ; k}\left(n ; Q_{t}^{*}\right)$ differs from $S_{2}\left(n ; Q_{t}^{*}\right)$ by the set of summation on the right hand side of (62). Namely, the summation on the right hand side of (62) is considered with respect to all those elements of the set $I$ such that there exist $l_{1}, \ldots, l_{k} \in\{1,2,3,4\}$ for which $\Gamma^{\left(l_{s}\right)}<\left(\begin{array}{c}\varepsilon n \\ 2\end{array}\right)$ and $\Gamma^{(r)} \geq\left(\begin{array}{c}\varepsilon n \\ 2\end{array}\right)$, where $r \in\{1,2,3,4\} \backslash\left\{l_{1}, \ldots, l_{k}\right\}, s=1, \ldots, k, k=1, \ldots, 4$.

For each $k=1, \ldots, 4$, we represent $S_{2 ; k}\left(n ; Q_{t}^{*}\right)$ in the following form:

$$
S_{2 ; k}\left(n ; Q_{t}^{*}\right)=\sum_{1 \leq t_{1}<\cdots<t_{k} \leq 4} S_{2 ; k ; t_{1}, \ldots, t_{k}}\left(n ; Q_{t}^{*}\right),
$$

where $S_{2 ; k ; t_{1}, \ldots, t_{k}}\left(n ; Q_{t}^{*}\right)$ denotes the sum of all terms of $S_{2 ; k}\left(n ; Q_{t}^{*}\right)$ for which

$$
\Gamma^{\left(t_{l}\right)}<\left(\begin{array}{c}
\varepsilon n \\
2
\end{array}\right), \quad l=1, \ldots, k, \quad \Gamma^{\left(t^{\prime}\right)} \geq\left(\begin{array}{c}
\varepsilon n \\
2
\end{array}\right), \quad t^{\prime} \in\{1,2,3,4\} \backslash\left\{t_{1}, \ldots, t_{k}\right\} .
$$

We show that, for all $k=1$,

$$
S_{2 ; k}\left(n ; Q_{t}^{*}\right) \leq a_{10} 4^{n}(1+o(1))
$$

as $n \rightarrow \infty$.

Using (3), (12), and (35) and recalling the definition of the sum

$$
\begin{gathered}
S_{2 ; 1 ; 1}\left(n ; Q_{t}^{*}\right) \\
\left(S_{2 ; 1 ; 4}\left(n ; Q_{t}^{*}\right)\right),
\end{gathered}
$$

we obtain

$$
\begin{gathered}
S_{2 ; 1 ; 1}\left(n ; Q_{t}^{*}\right) \leq a_{11} 2^{n} S_{2 ; 1 ; 1}(n ; 1) \\
\left(S_{2 ; 1 ; 4}\left(n ; Q_{t}^{*}\right) \leq a_{11} 2^{n} S_{2 ; 1 ; 4}(n ; 1)\right) .
\end{gathered}
$$


The inequality $\Gamma^{(1)}<\left(\begin{array}{c}\varepsilon n \\ 2\end{array}\right)\left(\Gamma^{(4)}<\left(\begin{array}{c}\varepsilon n \\ 2\end{array}\right)\right)$ and relation (13) ((16)) imply that all parameters $i, l$, and $h$ involved in forming the sum $S\left(n ; Q_{t}^{*}\right)$ (see (62) do not exceed $\varepsilon n$. Then the polynomial theorem implies that, for $k=1$,

$$
\begin{gathered}
S_{2 ; k ; 1}(n ; 1) \leq \exp \left\{\sigma_{1}(\varepsilon) n\right\} \\
\left(S_{2 ; k ; 4}(n ; 1) \leq \exp \left\{\sigma_{2}(\varepsilon) n\right\}\right)
\end{gathered}
$$

as $n \rightarrow \infty$, where $\sigma_{r}\left(\varepsilon_{0}\right) \rightarrow 0$ as $\varepsilon_{0} \rightarrow 0$ for $r=1,2, \ldots$.

Taking into account (70) and (72) ((71) and (73)), we prove the following bound:

$$
\begin{gathered}
S_{2 ; 1 ; 1}\left(n ; Q_{t}^{*}\right) \leq a_{11} 2^{n} \exp \left\{\sigma_{1}(\varepsilon) n\right\} \\
\left(S_{2 ; 1 ; 4}\left(n ; Q_{t}^{*}\right) \leq a_{11} 2^{n} \exp \left\{\sigma_{2}(\varepsilon) n\right\}\right) .
\end{gathered}
$$

Further, the inequalities $\Gamma^{(2)}<\left(\begin{array}{c}\varepsilon n \\ 2\end{array}\right)$ and $t-l=i+h \geq 2$ (see Lemma 2.2) and relation (14) allow one to rewrite the sum $S_{2 ; 1 ; 2}\left(n ; Q_{t}^{*}\right)$ as follows:

$$
S_{2 ; 1 ; 2}\left(n ; Q_{t}^{*}\right)=\sum_{l=1}^{3} S_{2 ; 1 ; 2}^{(l)}\left(n ; Q_{t}^{*}\right)
$$

where

$$
S_{2 ; 1 ; 2}^{(l)}\left(n ; Q_{t}^{*}\right)=\sum_{t=3}^{n}\left(\begin{array}{l}
n \\
t
\end{array}\right) \sum_{q \in \mathbb{R}_{l}}\left(\begin{array}{l}
t \\
q
\end{array}\right) \sum_{i+h=q} \frac{q !}{i ! h !} Q_{t}^{*}, \quad l=1,2,3 .
$$

The closed intervals $R_{l}, l=1,2,3$, with integer end points are given by

$$
R_{1}=\left[2 ;\left[\sqrt{\frac{\varepsilon^{\prime} n}{\ln n}}\right]\right], \quad R_{2}=\left[\left[\sqrt{\frac{\varepsilon^{\prime} n}{\ln n}}\right]+1 ;\left[\varepsilon^{\prime \prime} n\right]\right], \quad R_{3}=\left[\left[\varepsilon^{\prime \prime} n\right]+1 ; t\right],
$$

where $\varepsilon^{\prime}$ and $\varepsilon^{\prime \prime}$ are fixed positive numbers such that $0<\varepsilon^{\prime}, \varepsilon^{\prime \prime}<1$.

Taking into account (12), we have for $q \in \mathbb{R}_{1}$

$$
Q_{t}^{*} \leq 3^{T}\left(1-2 p_{\min }\left(\begin{array}{c}
q \\
2
\end{array}\right)\left(1+O\left(p_{\min }\left(\begin{array}{l}
q \\
2
\end{array}\right)\right)\right)+2 \exp \left\{-3 p_{\min }\left(\begin{array}{c}
\varepsilon n \\
2
\end{array}\right)\right\}\right)^{T}
$$

as $n \rightarrow \infty$. Bound (77) together with relations (3) and (35) implies

$$
Q_{t}^{*} \leq a_{12} 3^{\frac{\ln 2}{\ln 3} n}\left(1-\frac{q c \ln n}{n}\left(1+O\left(\varepsilon^{\prime}\right)\right)+2 n^{-\frac{3}{2} c \varepsilon^{2} n(1+o(1))}\right)^{\frac{\ln 2}{\ln 3} n}
$$

as $n \rightarrow \infty$.

Thus, if $q \in \mathbb{R}_{1}$, then

$$
Q_{t}^{*} \leq a_{13} 2^{n} \exp \left\{\frac{2 \ln 2(1+o(1))}{n^{\frac{3}{2} c \varepsilon^{2} n(1+o(1))-1} \ln 3}\right\}\left(\frac{1}{n^{c \frac{\ln 2}{\ln 3}\left(1+o(1)+O\left(\varepsilon^{\prime}\right)\right)}}\right)^{q}
$$

as $n \rightarrow \infty$. The definition of the sum $S_{2 ; 1 ; 2}^{(1)}\left(n ; Q_{t}^{*}\right)$ and relation (78) imply

$$
S_{2 ; 1 ; 2}^{(1)}\left(n ; Q_{t}^{*}\right) \leq a_{14} 4^{n} \sum_{q=0}^{\infty} \frac{1}{q !}\left(\frac{2}{n^{c \frac{\ln 2}{\ln 3}\left(1+O\left(\varepsilon^{\prime}\right)\right)-1}}\right)^{q}
$$

as $n \rightarrow \infty$. Using equality (12), we get for $q \in \mathbb{R}_{2}$

$$
Q_{t}^{*} \leq\left(1+2 \exp \left\{-3 p_{\min }\left(\begin{array}{c}
\left.\sqrt{\frac{\varepsilon^{\prime} n}{\ln n}}\right]+1 \\
2
\end{array}\right)\right\}+6 \exp \left\{-3 p_{\min }\left(\begin{array}{c}
\varepsilon n \\
2
\end{array}\right)\right\}\right)^{T} .
$$


Now we apply (3), (35), and (80) to prove that

$$
Q_{t}^{*} \leq\left(1+\frac{2}{e^{\frac{3}{2} c \varepsilon^{\prime}(1+o(1))}}+\frac{6}{n^{\frac{3}{2} c \varepsilon^{2} n(1+o(1))}}\right)^{\frac{\ln 2}{\ln 3} n+m_{0}}
$$

as $n \rightarrow \infty$ for $q \in \mathbb{R}_{2}$.

Recalling the definition of the $\operatorname{sum} S_{2 ; 1 ; 2}^{(2)}\left(n ; Q_{t}^{*}\right)$ we deduce from the polynomial formula and bound (81) that

$$
S_{2 ; 1 ; 2}^{(2)}\left(n ; Q_{t}^{*}\right) \leq a_{15} 2^{n} e^{\sigma_{3}\left(\varepsilon^{\prime \prime}\right) n}\left(1+\frac{2}{e^{\frac{3}{2} c \varepsilon^{\prime}(1+o(1))}}+\frac{6}{n^{\frac{3}{2} c \varepsilon^{2} n(1+o(1))}}\right)^{\frac{\ln 2}{\ln 3} n}
$$

as $n \rightarrow \infty$. For $q \in \mathbb{R}_{3}$, we take into account equality (12) and similarly to (82) find that

$$
Q_{t}^{*} \leq\left(1+\frac{2}{n^{\frac{3}{2} c\left(\varepsilon^{\prime \prime}\right)^{2} n(1+o(1))}}+\frac{6}{n^{\frac{3}{2} c \varepsilon^{2} n(1+o(1))}}\right)^{\frac{\ln 2}{\ln 3 n+m_{0}}}
$$

as $n \rightarrow \infty$. Again using the polynomial formula together with bound (83) we obtain

$$
S_{2 ; 1 ; 2}^{(3)}\left(n ; Q_{t}^{*}\right) \leq a_{16} 4^{n}
$$

as $n \rightarrow \infty$. Combining (76), (779), (82), and (84) we get

$$
S_{2 ; 1 ; 2}\left(n ; Q_{t}^{*}\right) \leq a_{17} 4^{n}(1+o(1))
$$

as $n \rightarrow \infty$. Further, let $\Gamma^{(3)}<\left(\begin{array}{c}\varepsilon n \\ 2\end{array}\right), t-i \geq 2$. Then

$$
S_{2 ; 1 ; 3}\left(n ; Q_{t}^{*}\right)=\sum_{l=1}^{3} S_{2 ; 1 ; 3}^{(l)}\left(n ; Q_{t}^{*}\right)
$$

where

$$
S_{2 ; 1 ; 3}^{(l)}\left(n ; Q_{t}^{*}\right)=\sum_{t=3}^{n}\left(\begin{array}{l}
n \\
t
\end{array}\right) \sum_{q \in \mathbb{R}_{l}}\left(\begin{array}{l}
t \\
q
\end{array}\right) \sum_{l+h=q} \frac{q !}{l ! h !} Q_{t}^{*}, \quad l=1,2,3 .
$$

This representation together with (15) allows one to prove similarly to (85) that

$$
S_{2 ; 1 ; 3}\left(n ; Q_{t}^{*}\right) \leq a_{18} 4^{n}(1+o(1))
$$

as $n \rightarrow \infty$. Now we derive inequality (69) with $k=1$ from relations (68), (74), (75), (85), and (86).

Next we show that, for $k=2$,

$$
S_{2 ; k}\left(n ; Q_{t}^{*}\right) \leq a_{19} 5^{\frac{\ln 2}{\ln 3} n} e^{\sigma_{4}(\varepsilon) n}
$$

as $n \rightarrow \infty$.

Indeed, relations (3), (12), (35), and (68) imply that, for $k=2$,

$$
S_{2 ; k}\left(n ; Q_{t}^{*}\right) \leq a_{20} 5^{\frac{\ln 2}{\ln 3} n}\left(\sum_{1 \leq t_{1}<t_{2} \leq 4} S_{2 ; k ; t_{1}, t_{2}}(n ; 1)\right)
$$

as $n \rightarrow \infty$. Then the inequalities $\Gamma^{\left(t_{1}\right)}<\left(\begin{array}{c}\varepsilon n \\ 2\end{array}\right)$ and $\Gamma^{\left(t_{2}\right)}<\left(\begin{array}{c}\varepsilon n \\ 2\end{array}\right)$, where $1 \leq t_{1}<t_{2} \leq 4$, together with (13)-(16) yield that the parameters $i, l$, and $h$ on the right hand side of (62) do not exceed $\varepsilon n$. This, in turn, implies the bound

$$
\max _{1 \leq t_{1}<t_{2} \leq 4} S_{2 ; 2 ; t_{1}, t_{2}}(n ; 1) \leq a_{21} e^{\sigma_{5}(\varepsilon) n},
$$


whence

$$
\sum_{1 \leq t_{1}<t_{2} \leq 4} S_{2 ; 2 ; t_{1}, t_{2}}(n ; 1) \leq a_{22} e^{\sigma_{5}(\varepsilon) n} .
$$

Now inequality (87) with $k=2$ follows from (88) and (89).

Next we prove that

$$
S_{2 ; k}\left(n ; Q_{t}^{*}\right) \leq a_{23} 7^{\frac{\ln 2}{\ln 3} n} e^{\sigma_{6}(\varepsilon) n}
$$

for $k=3$ as $n \rightarrow \infty$.

Indeed, relations (3), (12), (35), and (68) imply that

$$
S_{2 ; k}\left(n ; Q_{t}^{*}\right) \leq a_{24} 7^{\frac{\ln 2}{\ln 3} n}\left(\sum_{1 \leq t_{1}<t_{2}<t_{3} \leq 4} S_{2 ; k ; t_{1}, t_{2}, t_{3}}(n ; 1)\right)
$$

for $k=3$ as $n \rightarrow \infty$.

The bound

$$
\sum_{1 \leq t_{1}<t_{2}<t_{3} \leq 4} S_{2 ; 3 ; t_{1}, t_{2}, t_{3}}(n ; 1) \leq a_{25} e^{\sigma_{7}(\varepsilon) n}
$$

is proved analogously to (89). Then (91) and (92) prove inequality (90) for $k=3$.

Finally, we show that

$$
S_{2 ; k}\left(n ; Q_{t}^{*}\right) \leq a_{26} 4^{n}(1+o(1))
$$

for $k=4$ as $n \rightarrow \infty$. Note that the parameters $i, l$, and $h$ on the right hand side of (62) are such that

$$
\max (i, l, h)<\varepsilon n .
$$

Inequality (94) follows from $\Gamma^{(r)}<\left(\begin{array}{c}\varepsilon n \\ 2\end{array}\right), r=1, \ldots, 4$, in view of (13)-(16).

In particular, inequality (94) allows one to represent $S_{2 ; 4}\left(n ; Q_{t}^{*}\right)$ in the following form:

$$
S_{2 ; 4}\left(n ; Q_{t}^{*}\right)=\sum_{p=1}^{4} S_{2 ; 4}^{(p)}\left(n ; Q_{t}^{*}\right)
$$

where

$$
S_{2 ; 4}^{(p)}\left(n ; Q_{t}^{*}\right)=\sum_{t \in \mathbb{R}_{p}}\left(\begin{array}{l}
n \\
t
\end{array}\right) \sum_{i+l+h=t} \frac{t !}{i ! l ! h !} Q_{t}^{*}, \quad p=1, \ldots, 4 .
$$

The closed intervals $\mathbb{R}_{p}, p=1, \ldots, 4$, whose end points are integers, are equal to

$$
\begin{gathered}
R_{1}=[3 ; 7], \quad R_{2}=\left[8 ;\left[\frac{n}{\ln ^{2} n}\right]\right], \quad R_{3}=\left[\left[\frac{n}{\ln ^{2} n}\right]+1 ;\left[\frac{\delta n}{\ln n}\right]\right], \\
R_{4}=\left[\left[\frac{\delta n}{\ln n}\right]+1 ;[\varepsilon n]\right]
\end{gathered}
$$

where $\delta$ is a constant such that $0<\delta<\frac{2}{3 a_{2}}$.

We show that

$$
S_{2 ; 4}^{(1)}\left(n ; Q_{t}^{*}\right) \leq a_{27} 4^{n}(1+o(1))
$$

as $n \rightarrow \infty$. If $t=3$, the inequalities $i+l \geq 1, t-i \geq 2$, and $t-l \geq 2$ (see Lemma 2.2) imply that $i \in\{0,1\}, l \in\{0,1\}$, and $i+l \neq 0$. Further, we consider separately all possible combinations of the parameters $i$ and $l$. 
1. If $i=1$ and $l=0$ or $i=0$ and $l=1$, then we derive from (12) (16) that

$$
\begin{aligned}
\left(\begin{array}{l}
n \\
3
\end{array}\right) & \sum_{i+l+h=3} \frac{3 !}{i ! l ! h !} \prod_{\mu=1}^{T}\left(1+2\left(\sum_{r=1}^{4}\left(1-3 p_{\mu}\right)^{\Gamma^{(r)}}\right)\right) \\
= & \left(\begin{array}{l}
n \\
3
\end{array}\right) \prod_{\mu=1}^{T}\left(1+4\left(1-3 p_{\mu}\right)^{3}+2\left(1-3 p_{\mu}\right)^{2}+2\left(1-3 p_{\mu}\right)\right) .
\end{aligned}
$$

In view of (3), (35), and (98) we deduce that

$$
\left(\begin{array}{l}
n \\
3
\end{array}\right) \sum_{i+l+h=3} \frac{3 !}{i ! l ! h !} \prod_{\mu=1}^{T}\left(1+2\left(\sum_{r=1}^{4}\left(1-3 p_{\mu}\right)^{\Gamma^{(r)}}\right)\right) \leq \frac{a_{28} 4^{n}}{n^{\frac{6 c \ln 2}{\ln 3}(1+o(1))-3}}
$$

as $n \rightarrow \infty$.

2. If $i=1$ and $l=1$, then similarly to the proof of (99) we obtain

$$
\left(\begin{array}{l}
n \\
3
\end{array}\right) \sum_{i+l+h=3} \frac{3 !}{i ! l ! h !} \prod_{\mu=1}^{T}\left(1+2\left(\sum_{r=1}^{4}\left(1-3 p_{\mu}\right)^{\Gamma^{(r)}}\right)\right) \leq \frac{a_{29} 4^{n}}{n^{\frac{4 c \ln 2}{\ln 3}(1+o(1))-3}}
$$

as $n \rightarrow \infty$.

With the help of relations (99) and (100) we obtain that, for $t=3$,

$$
\left(\begin{array}{l}
n \\
t
\end{array}\right) \sum_{i+l+h=t} \frac{t !}{i ! l ! h !} \prod_{\mu=1}^{T}\left(1+2\left(\sum_{r=1}^{4}\left(1-3 p_{\mu}\right)^{\Gamma^{(r)}}\right)\right) \leq \frac{a_{30} 4^{n}}{n^{a_{31}(1+o(1))}}
$$

as $n \rightarrow \infty$. Now one can easily check that bound (101) holds for $t=4, \ldots, 7$, whence we derive inequality (97).

Then we show that

$$
S_{2 ; 4}^{(2)}\left(n ; Q_{t}^{*}\right) \leq a_{32} 4^{n} \sum_{t=0}^{\infty} \frac{1}{t !}\left(\frac{3}{n^{c \frac{\ln 2}{\ln 3}(1+o(1))-1}}\right)^{t}
$$

as $n \rightarrow \infty$.

Put $t_{p, \min }=\min _{t \in \mathbb{R}_{p}} t$ and $t_{p, \max }=\max _{t \in \mathbb{R}_{p}} t$ for $p=2,3$.

Taking into account relation (12) and Lemma 3.4 we get for $t \in \mathbb{R}_{p}, p=2,3$, that

$$
\begin{aligned}
Q_{t}^{*} & \leq\left(1+2\left(1+\left(1-3 p_{\min }\right)^{\frac{t}{4}\left(\frac{t}{2}-1\right)}+2\left(1-3 p_{\min }\right)^{\left(\frac{t}{2}-1\right)}\right)\right)^{T} \\
& \leq\left(9-3 p_{\min } t\left(1-\frac{2}{t_{p, \min }}\right) H_{t}\right)^{T}
\end{aligned}
$$

where

$$
H_{t}=\frac{t_{p, \min }}{4}+2-3 p_{\min }\left(\frac{t_{p, \max }}{2}-1\right)\left(\frac{1}{2}\left(\frac{t_{p, \min }}{4}\right)^{2}+1\right) .
$$

Using (3), (35), and (103) (for $p=2$ ), we get, for $t \in \mathbb{R}_{2}$,

$$
Q_{t}^{*} \leq a_{33} 4^{n}\left(\frac{1}{n^{c \frac{\ln 2}{\ln 3}(1+o(1))}}\right)^{t}
$$

as $n \rightarrow \infty$. The definition of $S_{2 ; 4}^{(2)}\left(n ; Q_{t}^{*}\right)$ and inequality (104) complete the proof of (102).

Next we prove that

$$
S_{2 ; 4}^{(3)}\left(n ; Q_{t}^{*}\right) \leq a_{34} 4^{n}\left(\frac{3 \ln ^{2} n}{n^{c \frac{\ln 2}{\ln 3}\left(1-\frac{3 c \delta}{2}\right)(1+o(1))}}\right)^{\frac{n}{\ln ^{2} n}} \sum_{t=0}^{\infty}\left(\frac{3 \ln ^{2} n}{n^{c \frac{\ln 2}{\ln 3}\left(1-\frac{3 c \delta}{2}\right)(1+o(1))}}\right)^{t}
$$


as $n \rightarrow \infty$. Taking into account (3), (35), and (103) (for $p=3$ ), we obtain, for $t \in \mathbb{R}_{3}$,

$$
Q_{t}^{*} \leq a_{35} 4^{n}\left(\frac{1}{n^{c \frac{\ln 2}{\ln 3}\left(1-\frac{3 c \delta}{2}\right)(1+o(1))}}\right)^{t}
$$

as $n \rightarrow \infty$. Thus bound (105) follows from (96) and (106).

Then we show that

$$
S_{2 ; 4}^{(4)}\left(n ; Q_{t}^{*}\right) \leq a_{36} 2^{n} \exp \left\{\sigma_{8}(\varepsilon) n\right\}\left(1+\frac{2}{\exp \left\{\frac{3}{2} c \delta(1+o(1))\right\}}\right)^{\frac{\ln 2}{\ln 3} n}
$$

as $n \rightarrow \infty$. Relations (3) and (35) together with Lemma 3.4 imply that

$$
Q_{t}^{*} \leq a_{37} 2^{n}\left(1+\frac{2}{\exp \left\{\frac{3}{2} c \delta(1+o(1))\right\}}\right)^{\frac{\ln 2}{\ln 3} n}
$$

as $n \rightarrow \infty$. Relation (94) proves that

$$
S_{2 ; 4}^{(4)}(n ; 1) \leq a_{38} n^{1 / 2} \exp \left\{\sigma_{8}(\varepsilon) n\right\}
$$

in view of the polynomial formula. Considering (96), (109), and (108) we get inequality (107).

Combining (95), (97), (102), (105), and (107) we obtain (93).

For $S_{2}\left(n ; Q_{t}^{*}\right)$, relations (67), (69), (87), (90), and (93) imply

$$
S_{2}\left(n ; Q_{t}^{*}\right) \leq a_{39} 4^{n}(1+o(1))
$$

as $n \rightarrow \infty$. Now $S\left(n ; Q_{t}^{*}\right) \leq a_{40} 4^{n}(1+o(1))$ as $n \rightarrow \infty$ by (63), (66), and (110). Thus bound (60) holds in view of relation (61). Inequalities (59) and (60) prove (55).

Summarizing, if (35) holds, then (54) and (55) hold as well. This together with (56) allows us to conclude that relation (53) holds, too. In turn, this contradicts the property that, with probability approaching zero as $n \rightarrow \infty$, there exists a solution of system (1) that belongs to the set $M_{n}$.

\section{Proof of Theorem 1.2}

Proof. Theorem 1.2 follows from (41), (44), (47), and (51).

EXAmples TO TheOREM 1.2

\begin{tabular}{|c|c|c|c|c|}
\hline & 1. & 2. & 3. & 4. \\
\hline$\varepsilon_{1}$ & 0.1 & 0.05 & 0.05 & 0.01 \\
\hline$\varepsilon_{2}$ & 0.02 & 0.01 & 0.01 & 0.01 \\
\hline$c$ & 5 & 10 & 10 & 100 \\
\hline$\gamma_{0}$ & 0.7 & 1 & 1 & 1 \\
\hline$\gamma_{1}$ & 0.5 & 0.4 & 0.4 & 1 \\
\hline$n$ & 500 & 1000 & 1000 & 10000 \\
\hline$A_{n}$ & $\ln n$ & $\ln n$ & $\ln \ln n$ & $\sqrt{\ln n}$ \\
\hline$Z_{1}$ & $1.1171 \times 10^{-3}$ & $5.1482 \times 10^{-4}$ & $1.1217 \times 10^{-1}$ & $3.5646 \times 10^{-2}$ \\
\hline
\end{tabular}




\section{EXAMPles TO THEOREM 1.3}

\begin{tabular}{|c|c|c|c|c|}
\hline & 1. & 2. & 3. & 4. \\
\hline$\varepsilon_{1}$ & 0.1 & 0.05 & 0.05 & 0.01 \\
\hline$\varepsilon_{2}$ & 0.02 & 0.01 & 0.01 & 0.01 \\
\hline$c$ & 5 & 10 & 10 & 100 \\
\hline$\beta_{0}$ & 0.7 & 1 & 1 & 1 \\
\hline$\beta_{1}$ & 0.5 & 0.4 & 0.4 & 1 \\
\hline$n$ & 500 & 1000 & 1000 & 10000 \\
\hline$A_{n}$ & $\ln n$ & $\ln n$ & $\ln \ln n$ & $\sqrt{\ln n}$ \\
\hline$a_{1}$ & 10 & 15 & 50 & 100 \\
\hline$\alpha$ & 0.2 & 0.1 & 0.2 & 0.23 \\
\hline$Z_{2}$ & $2.2747 \times 10^{-1}$ & $6.8333 \times 10^{-1}$ & $1.1965 \times 10^{-1}$ & $3.9291 \times 10^{-2}$ \\
\hline
\end{tabular}

\section{Proof of Theorem 1.3}

The assumptions of Theorem 1.3 imply that the terms $D_{1}, D_{2}$, and $D_{3}$ on the right hand side of relation (51) are such that $D_{1} \leq D_{1}^{\prime}, D_{2} \leq D_{2}^{\prime}$, and $D_{3} \leq D_{3}^{\prime}$ (this can be proved similarly to the proof of inequalities (41), (44), and (47), respectively), where $D_{1}^{\prime}=e / n^{2 \alpha}$,

$$
D_{2}^{\prime}=2^{n \sigma\left(\varepsilon_{2}\right)}\left(\frac{1}{3}+\frac{2}{3 e^{\frac{3}{2} \beta_{1}}}\right)^{n \frac{\ln 2}{\ln 3}}, \quad D_{3}^{\prime}=\left(\frac{\exp \left\{\frac{2}{n^{\frac{3}{2} n c \varepsilon_{2}^{2}}}\right\}}{3}\right)^{A_{n}} \exp \left\{\frac{2 \ln 2}{n^{\frac{3}{2} n c \varepsilon_{2}^{2}-1} \ln 3}\right\} .
$$

The above bounds prove Theorem 1.3 .

\section{CONCLUSION}

A necessary and sufficient condition is found showing the probability of the random event that a second order system of nonlinear random equations over the field $\mathbf{G F}(3)$ has a solution that belongs to a given set of vectors (Theorem 1.1). The condition is given in terms of the number of equations and number of unknowns.

Under various assumptions concerning the parameter $c$, defined by equality (3), several bounds are found for the above probability (Theorems 1.2 and 1.3). Some examples are given for Theorems 1.2 and 1.3

\section{BIBLIOGRAPHY}

1. V. A. Kopyttsev and V. G. Mikhailov, Poisson-type theorems for the number of special solutions of a random linear inclusion, Diskret. Matem. 22 (2010), no. 2, 3-21; English transl. in Discrete Math. Appl. 22 (2010), no. 2, 191-211. MR2730124 (2011m:60216)

2. V. I. Masol and L. A. Romashova, Uniqueness conditions for the solution of an inhomogeneous system of nonlinear random equations over the field GF(3), Kibernet. Sistem. Analiz (2010), no. 2, 23-36; English transl. in Cybernet. Systems Anal. 46 (2010), no. 2, 185-199. MR2921497

3. K. A. Rybnikov, Introduction to Combinatorial Analysis, second edition, Moscow University, Moscow, 1985. (Russian) MR812275 (87b:05002)

4. W. Feller, An Introduction to Probability Theory and its Applications, third edition, vol. 1, John Wiley \& Sons, Inc., New York-London-Sydney, 1968. MR0228020 (37:3604)

5. A. N. Shiryaev, Problems in Probability, "MCNMO", Moscow, 2006; English transl., Springer, New York, 2012. MR 2961901 
Department of Probability Theory, Statistics, and Actuarial Mathematics, Faculty for Mechanics and Mathematics, National Taras Shevchenko University, Academician Glushkov Avenue, 4E, Kiev 03127, Ukraine

Department of Probability Theory, Statistics, and Actuarial Mathematics, Faculty for Mechanics and Mathematics, National Taras Shevchenko University, Academician Glushkov Avenue, 4E, Kiev 03127, Ukraine

E-mail address: deezee@ukr.net

Received 04/JUL/2011

Translated by S. KVASKO 